\title{
Spliceosome-associated protein 130: a novel biomarker for idiopathic pulmonary fibrosis
}

\author{
Kaixiong Liu ${ }^{1,2}$, Dong Liu ${ }^{1,2}$, Yun Feng ${ }^{1,2}$, Hongying Zhang ${ }^{3}$, Dunhuan Zeng ${ }^{4}$, Qinhua Liu ${ }^{5}$ Jieming Qu ${ }^{1,2}$ \\ ${ }^{1}$ Department of Pulmonary and Critical Care Medicine, Ruijin Hospital, Shanghai Jiao Tong University School of Medicine, Shanghai, China; \\ ${ }^{2}$ Institute of Respiratory Diseases, School of Medicine, Shanghai Jiao Tong University, Shanghai, China; ${ }^{3}$ Department of Respiratory Medicine, \\ Fuzhou Pulmonary Hospital, Fuzhou, China; ${ }^{4}$ Department of Pulmonary and Critical Care Medicine, the First Affiliated Hospital, Fujian Medical \\ University, Fuzhou, China; ${ }^{5}$ Department of Respiratory disease, Fujian Geriatric Hospital, Fuzhou, China \\ Contributions: (I) Conception and design: K Liu, J Qu; (II) Administrative support: J Qu; (III) Provision of study materials or patients: K Liu, H \\ Zhang, D Zeng, Q Liu; (IV) Collection and assembly of data: K Liu, H Zhang, D Zeng, Q Liu; (V) Data analysis and interpretation: K Liu, D Liu; (VI) \\ Manuscript writing: All authors; (VII) Final approval of manuscript: All authors. \\ Correspondence to: Jieming Qu. Department of Pulmonary and Critical Care Medicine, Ruijin Hospital, Shanghai Jiao Tong University School of \\ Medicine, 197 Ruijin 2nd Road, Huangpu District, Shanghai, China. Email: jmqu0906@163.com.
}

Background: Spliceosome-associated protein 130 (SAP130), a novel danger-associated molecular pattern (DAMP), is involved in inflammatory disease. However, no data are available about SAP130 in idiopathic pulmonary fibrosis (IPF). Our study aimed to investigate SAP130 in the serum and lung tissue of patients with IPF and to determine its clinical significance.

Methods: SAP130 levels in the serum of 83 IPF patients and 38 healthy subjects were measured. Additionally, immunohistochemical staining for SAP130 was performed in lung specimens of IPF patients and control subjects. Correlation between serum SAP130 levels and clinical parameters were investigated.

Results: Serum SAP130 levels were elevated in IPF patients compared with healthy controls. In parallel, the expression of SAP130 in lung tissue was elevated in IPF. SAP130 levels were higher in patients with acute exacerbation of idiopathic pulmonary fibrosis (AE-IPF) than patients with stable IPF $(\mathrm{P}=0.0144)$. The area under curve (AUC) of the ROC curve for the diagnosis of IPF was 0.944 (95\% CI, 0.810-0.997) for SAP130. The sensitivity (92.1\%) and specificity (69.9\%) were obtained for the cutoff value of $643.87 \mathrm{pg} / \mathrm{mL}$. In patients with stable IPF, the SAP130 level correlated positively with fibrosis on high-resolution CT (HRCT) $(\mathrm{r}=0.4164, \mathrm{P}=0.0029)$ and serum KL-6 ( $\mathrm{r}=0.4564, \mathrm{P}=0.0010)$, and inversely with FEV1 ( $\mathrm{r}=-0.3562, \mathrm{P}=0.0120)$ and DLCO ( $\mathrm{r}=-0.5550, \mathrm{P}<0.0001)$. In patients with AE-IPF, the SAP130 level correlated positively with fibrosis ( $\mathrm{r}=0.3735, \mathrm{P}=0.0296)$ and ground-glass opacity $(\mathrm{r}=0.4697, \mathrm{P}=0.0051)$ on HRCT and serum Krebs von den Lungen $6(\mathrm{KL}-6)(\mathrm{r}=0.5470, \mathrm{P}=0.0008)$.

Conclusions: The study suggested that SAP130 was a potential noninvasive biomarker that correlates well with disease severity of IPF. A prospective, multicentre study is required to validate the clinical and pathophysiological utility of SAP130 in IPF.

Keywords: Idiopathic pulmonary fibrosis (IPF); spliceosome-associated protein 130 (SAP130); biomarkers; acute exacerbation

Submitted May 31, 2020. Accepted for publication Jun 28, 2020.

doi: $10.21037 /$ atm-20-4404

View this article at: http://dx.doi.org/10.21037/atm-20-4404 


\section{Introduction}

Idiopathic pulmonary fibrosis (IPF) is a chronic, progressive, devastating lung disease characterized by pulmonary insufficiency and diminished exercise capacity $(1,2)$.

The median survival is estimated at $3-5$ years after initial diagnosis. Each patient with IPF exhibits long periods of stability, a steady gradual decline, and/or periods of acute exacerbation. Acute exacerbation is defined as any acute respiratory event characterized by new bilateral ground glass opacification (GGO)/consolidation not fully explained by cardiac failure or fluid overload, which are associated with high morbidity and mortality $(3,4)$. Hence, biomarkers for early and accurate diagnosis of IPF, stratification of patients with respect to severity and predicting its prognosis, and monitoring therapy response are critical for clinical management.

A number of potential biomarkers have been identified, but none is prospectively validated for clinical practice (5). IPF-specific biomarkers should act as a surrogate reflecting alveolar epithelial cells, vascular and endothelial damage, aberrant fibrogenesis, fibroproliferation and matrix remodeling, as well as immune dysregulation. Some biomarkers were passively released from membranecompromised necrotic or secondary necrotic cells, or synthesized and released upon sensing of cellular damage or inflammation. Human IPF lung tissue exhibits heightened alveolar epithelial cell death including regulated/ programmed cell death (6). Some danger-associated molecular patterns (DAMPs) released from damaged or dying alveolar epithelial cells have been shown to be upregulated and capable of activating pattern recognition receptors in lung fibrosis. They played pivotal roles in the pathogenesis of lung fibrosis (7). The current treatment options for IPF, including lung transplantation and the recently approved Nintedanib and Pirfenidone, are limited and there is an urgent need for new therapeutic targets. Targeting the receptor and DAMPs by specific inhibitors in lung fibrosis may be a promising approach.

Spliceosome-associated protein 130 (SAP130), a subunit of histone deacetylase, is located in the nucleus in normal live cells but diffuses out of dying cells or damaged cells and is released to the extracellular milieu (8). SAP130 was recently demonstrated to be one of the novel DAMP and specifically binded to the Mincle (macrophage-inducible C-type lectin, CLEC4E) receptor, leading to trigger proinflammatory signaling under various infections and aseptic inflammation (9). Mincle is a transmembrane pattern recognition receptor that is highly expressed on activated myeloid cells and initiates spleen tyrosine kinase (Syk) signaling axis to transduce downstream signals to mediate the inflammatory response (10). SAP130 is increased in the intestinal mucosa and in the circulation of patients with Crohn's disease (11). SAP130 varied according to clinical activity and significantly correlated with disease severity of Crohn's disease (11). As SAP130 was released by damaged and dead cells, we hypothesized that serum SAP130 levels would be elevated in patients with IPF and would correlate with severity of disease. Until now, no data have been available on SAP130 expression in IPF. The present study was conducted to investigate SAP130 expression in the serum and lung of IPF patients and to determine its clinical significance. We present the following article in accordance with the STARD reporting checklist (available at http:// dx.doi.org/10.21037/atm-20-4404).

\section{Methods}

\section{Subjects}

Eighty-three IPF patients were included consecutively at the time of initial diagnosis at Ruijin Hospital of Shanghai Jiao Tong University, Fuzhou Pulmonary Hospital, First Affiliated Hospital of Fujian Medical University, and Fujian Geriatric Hospital between January 2017 and December 2018. The diagnosis of IPF was according to the American Thoracic Society (ATS)/European Respiratory Society (ERS) criteria $(12,13)$. Clinically stable was defined as no disease exacerbations for at least three months. Diagnosis of acute exacerbation of idiopathic pulmonary fibrosis (AEIPF) was made according to the International Working Group Report diagnostic criterial (3). The exclusion criteria were presence of severe comorbidity at the time of diagnosis (liver cirrhosis, advanced malignancy or renal failure requiring haemodialysis). Age- and sex-matched healthy subjects served as controls and were recruited from the Health checkup center of Ruijin Hospital. These healthy subjects had no evidence of lung diseases on low-dose chest-computed tomography scans. All patients underwent clinical, radiographic, and physiologic assessment. Data concerning the general characteristics, 6-minute walk test (6MWT), pulmonary function test (PFT) results, and chest high-resolution CT (HRCT) of patients with stable IPF were collected at the initial visit. Patients with AE-IPF were followed for at least 1 year. Survival status was obtained from visits and telephone interviews. The study was 
conducted according to the principles of the Declaration of Helsinki (as revised in 2013). The study was approved by the ethics committee at each hospital. Informed consent was obtained from all subjects included in our study.

\section{Lung function test}

PFT was performed routinely to assess the functional exercise capacity in subjects with stable IPF at enrollment. Most patients with AE-IPF had extreme desaturation, which prevented most of them from undergoing PFT. The tests measured the forced expiratory volume in 1 second (FEV1), forced vital capacity (FVC), and carbon monoxide diffusing capacity (DLCO). All tests were performed using a Jaeger pulmonary function instrument (Germany) in accordance with the American Thoracic Society standards/ European Respiratory Society guidelines (14). The 6MWT was performed to assess the functional exercise capacity in IPF patients with stable conditions at the baseline study visit, as previously described (15).

\section{Chest HRCT}

Chest HRCT examinations were performed on $1.0 \mathrm{~mm}$ thick sections and for all patients at the time of diagnosis to determine lung abnormalities. The presence and extent of visual GGO and fibrosis (including reticulation and honeycombing) were determined visually in the upper, middle, and lower zones of each side based on the percentage of the lung field that showed each abnormality in each zone (estimated to the nearest $10 \%$ of parenchymal involvement) as previously described (16). The upper lung zone was defined as the area at and above the level of the carina; the lower lung zone was the area at and below the inferior pulmonary vein; and the middle zone was the area between the upper and lower zones (17). The scores were obtained by averaging the six lung zones. Two radiologists blinded to patient disease characteristics independently performed a visual assessment of lung abnormalities in each patient.

\section{Enzyme linked immunosorbent assay (ELISA)}

Human peripheral blood was obtained from IPF patients and healthy volunteers. Plasma specimens were obtained by centrifugation, aliquoted, and stored at $-80{ }^{\circ} \mathrm{C}$ until ELISA was performed. SAP130 and Krebs von den Lungen 6 (KL6) were measured in plasma specimens by ELISA according to the manufacturer's protocol (Human Splicing Factor 3B
Subunit 3 ELISA Kit; EK17556 SAB Biotechnology, and KL-6 ELISA kit; orb153677 Biorbyt Biotechnology). Each sample was tested in duplicate.

\section{Immunobistochemistry (IHC)}

SAP130 expression in lung tissues from 9 cases of IPF and from 6 matching control subjects was examined by IHC. Lung tissues from IPF patients were obtained from pulmonary explants remaining after lung transplantation. Additional non-fibrotic control lung tissues were obtained from patients who underwent surgery for benign nodules, harvesting morphologically normal lung tissues. Lung explant specimens from patients with IPF and normal control lungs were fixed with neutral-buffer formalin and embedded in paraffin. Sections were deparaffinized and stained with Anti-SAP130 antibodies (ab 111739, Abcam) followed by biotinylated goat anti-mouse IgG and avidin-HRP.

\section{Statistical analysis}

Data were expressed as means \pm standard deviations. Differences between the two groups were analyzed by the Fisher's exact test or Wilcoxon rank-sum test. Serum levels of SAP130 were analyzed using a receiver operating characteristic curve (ROC) curve to determine the appropriate cutoff level for identifying IPF patients and healthy controls, as well as patients with stable IPF and patients with AE-IPF. Spearman's rank correlations were calculated to determine the correlation between SAP 130 levels and various clinical and laboratory parameters. Statistical analysis was performed using SPSS 19.0 (SPSS, Chicago, IL, USA). Significant differences were defined as $\mathrm{P}<0.05$.

\section{Results}

\section{Patient characteristics}

A total of 83 IPF patients (49 patients with stable IPF and 34 patients with AE-IPF) and 38 healthy control individuals were enrolled. All subjects enrolled were Chinese. The demographics and main characteristics of IPF patients and healthy control individuals were summarized in Table 1. The demographic, clinical, and HRCT data in patients with stable IPF and patients with AE-IPF were shown in Table 2. There were no significant differences in gender, age, and smoking status between patients with IPF and controls. No significant difference in age, gender, and smoking status 
Table 1 Demographic and clinical characteristics of IPF patients and healthy control subjects

\begin{tabular}{lccc}
\hline Characteristics & IPF $(n=83)$ & Healthy control $(\mathrm{n}=38)$ & $\mathrm{P}$ value \\
\hline Men/women & $65 / 18$ & $25 / 13$ & 0.143 \\
Age, years (mean $\pm \mathrm{SD})$ & $66.90 \pm 0.85$ & & 0.345 \\
Smoking status & & 10 & 0.793 \\
Never-smoker & 20 & 25 & 0.653 \\
Former smoker & 58 & 3 & 0.701 \\
Current smoker & 5 & $1,686 \pm 49.80$ & $<0.0001$ \\
Serum KL-6 $(\mathrm{U} / \mathrm{mL})$ & $865.3 \pm 54.65$ & & \\
\hline
\end{tabular}

IPF, idiopathic pulmonary fibrosis; KL-6, Krebs von den Lungen 6.

Table 2 Demographic and clinical characteristics of patients with stable IPF and patients with AE-IPF

\begin{tabular}{|c|c|c|c|}
\hline Characteristics & Stable IPF $(n=49)$ & AE-IPF $(n=34)$ & $P$ value \\
\hline Men/women & $39 / 10$ & $26 / 8$ & 0.734 \\
\hline Age, years (mean $\pm S D$ ) & $66.39 \pm 1.26$ & $67.65 \pm 1.29$ & 0.471 \\
\hline \multicolumn{4}{|l|}{ Smoking status } \\
\hline Never-smoker & 13 & 7 & 0.534 \\
\hline 6MWD & $297.73 \pm 104.59$ & ND & - \\
\hline FEV1 (\% predicted) & $68.70 \pm 20.62$ & ND & - \\
\hline FVC (\% predicted) & $65.54 \pm 20.08$ & ND & - \\
\hline DLCO (\% predicted) & $44.93 \pm 19.10$ & ND & - \\
\hline \multicolumn{4}{|l|}{ CT visual score } \\
\hline Fibrosis Score (\%) & $18.86 \pm 1.182$ & $25.41 \pm 1.95$ & 0.003 \\
\hline Serum KL-6 (U/mL) & $1,496 \pm 59.57$ & $1,958 \pm 61.51$ & $<0.0001$ \\
\hline
\end{tabular}

ND, no detected; AE-IPF, acute exacerbation of idiopathic pulmonary fibrosis; 6MWD, 6-minute walk distance; FEV1, forced expiratory volume in 1; FVC, forced vital capacity; DLCO, carbon monoxide diffusing capacity; GGO, ground glass opacification.

were also observe red between the AE-IPF and stable IPF groups. Patients in the stable phase had a higher rate of being lost to follow-up, and no mortality analysis was performed. Because hospitalized patients with AE-IPF had better compliance, only 4 patients were lost to follow-up. Most follow-ups were longer than 1 year.

\section{Increased serum SAP130 levels in patients with IPF}

To investigate the association of SAP130 with IPF, we first compared SAP130 levels between 83 IPF patients and 38 healthy controls at study entry by using ELISA (Figure 1).
Serum SAP130 levels were significantly elevated in patients with IPF more than in control individuals $(824.2 \pm 29.84 v$ s. $413.8 \pm 19.77 \mathrm{pg} / \mathrm{mL}, \mathrm{P}<0.0001)$. Serum SAP130 levels in patients with AE-IPF were significantly higher than that in the stable IPF patients $(763.9 \pm 37.16 v s .911 .1 \pm 46.04 \mathrm{pg} / \mathrm{mL}$, $\mathrm{P}=0.0144)$. Serum KL-6 levels were significantly higher in patients with AE-IPF than in patients with stable IPF $(1,686 \pm 49.80$ vs. $865.3 \pm 54.65 \mathrm{U} / \mathrm{mL}, \mathrm{P}<0.0001)$.

\section{IHC}

ICH analyses showed that the expression of SAP130 in 

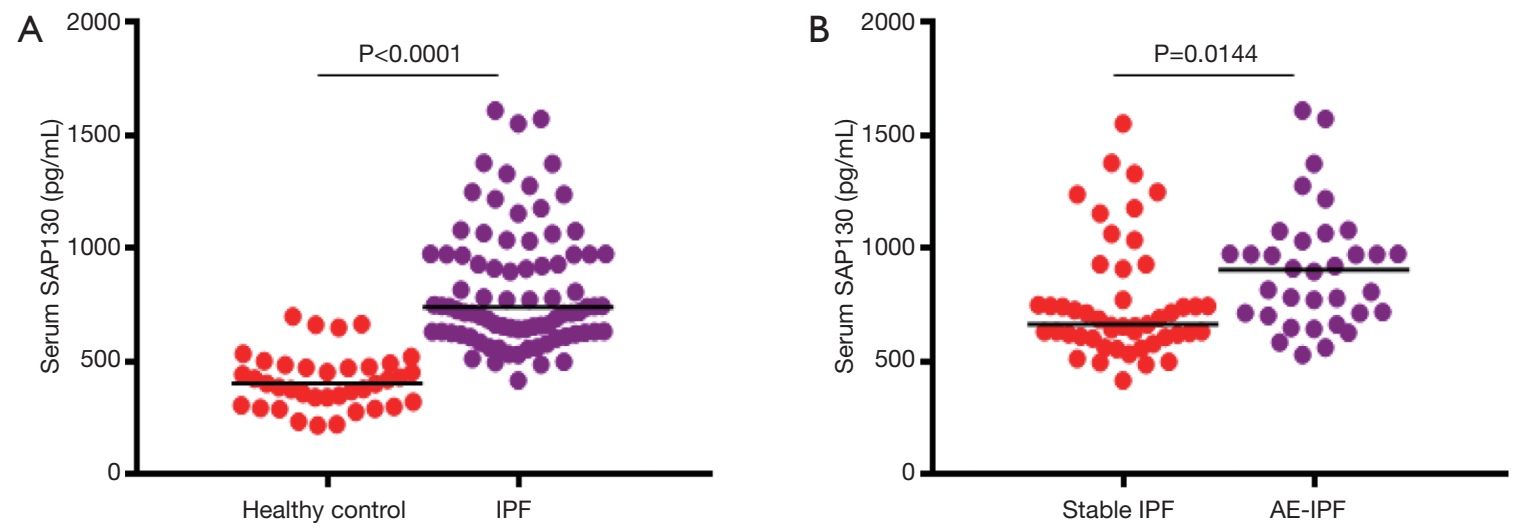

Figure 1 Baseline serum SAP130 levels. (A) Distribution of serum levels of SAP130 in IPF patients and control subjects. IPF patients showed significantly higher serum SAP130 levels than normal control subjects $(\mathrm{P}<0.0001)$. (B) Distribution of serum levels of SAP130 in patients with stable IPF and patients with AE-IPF. AE-IPF patients showed significantly higher serum SAP130 levels than stable IPF patients $(\mathrm{P}=0.0144)$. Scatter plots showing the baseline distribution. SAP130, spliceosome-associated protein 130; AE-IPF, acute exacerbation of idiopathic pulmonary fibrosis.

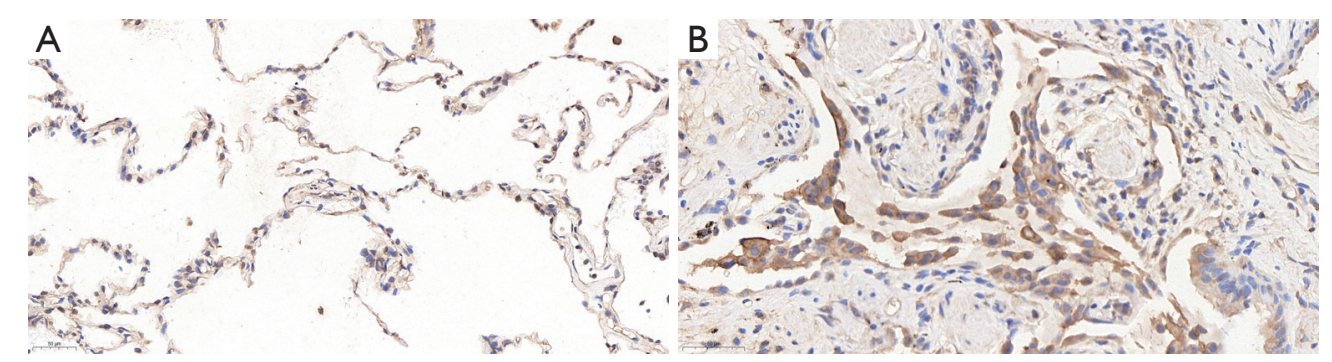

Figure 2 Expression of SAP130 in lung tissue of IPF patients and control subjects (IHC staining). (A) Representative image of control lung tissues (scale bar: $50 \mu \mathrm{m}$ ) showed SAP130 protein is expressed sparsely in normal alveolar structures. (B) Representative image of lung tissues of an IPF patient (scale bar: $50 \mu \mathrm{m}$ ) showed SAP130 protein is dramatically increased in IPF compared to control. SAP130 localizes diffusely to alveolar epithelial cells adjacent to the areas of mature fibrosis. SAP130, spliceosome-associated protein 130; IPF, idiopathic pulmonary fibrosis; IHC, immunohistochemistry.

lung tissue was significantly higher in IPF patients than in controls. SAP130 was sparsely expressed in normal alveolar tissue of control lungs (Figure $2 A$, scale bar $=20 \mu \mathrm{m}$; Figure $S 1 A$, scale bar $=100 \mu \mathrm{m}$ ). SAP130 localizes diffusely to the alveolar epithelial lining in the honeycomb space adjacent to the areas of mature fibrosis in IPF patients (Figure $2 B$, scale bar $=20 \mu \mathrm{m}$; Figure $S 1 B$, scale bar $=100 \mu \mathrm{m})$.

\section{Specificity and sensitivity of measurement of serum SAP130 levels}

The derived area under the ROC curve between IPF patients and healthy control subjects was 0.944 (95\% CI, 0.810-0.997) (Figure $3 A$ ). The optimal cutoff value of the serum SAP130 level was $643.87 \mathrm{pg} / \mathrm{mL}$. The sensitivity and specificity of
SAP130 were $92.1 \%$ and $69.9 \%$, respectively (Figure 3A). We also evaluated the sensitivity and specificity of serum SAP130 levels to distinguish AE-IPF from stable IPF based on ROC curves. The area under curve (AUC) of the ROC curve for the diagnosis of stable IPF versus AE-IPF diagnoses was 0.694 (95\% CI, 0.580-0.809) for SAP130 (Figure 3B). The sensitivity $(63.3 \%)$ and specificity $(67.6 \%)$ were obtained for the cutoff value of $741.46 \mathrm{pg} / \mathrm{mL}$ (Figure 3B).

\section{Correlation of SAP130 and clinical parameters in IPF patients}

We also determined whether serum SAP130 was associated with the severity of IPF (Figures 4,5). Spearman's analysis revealed that serum levels of SAP 130 in stable IPF patients 

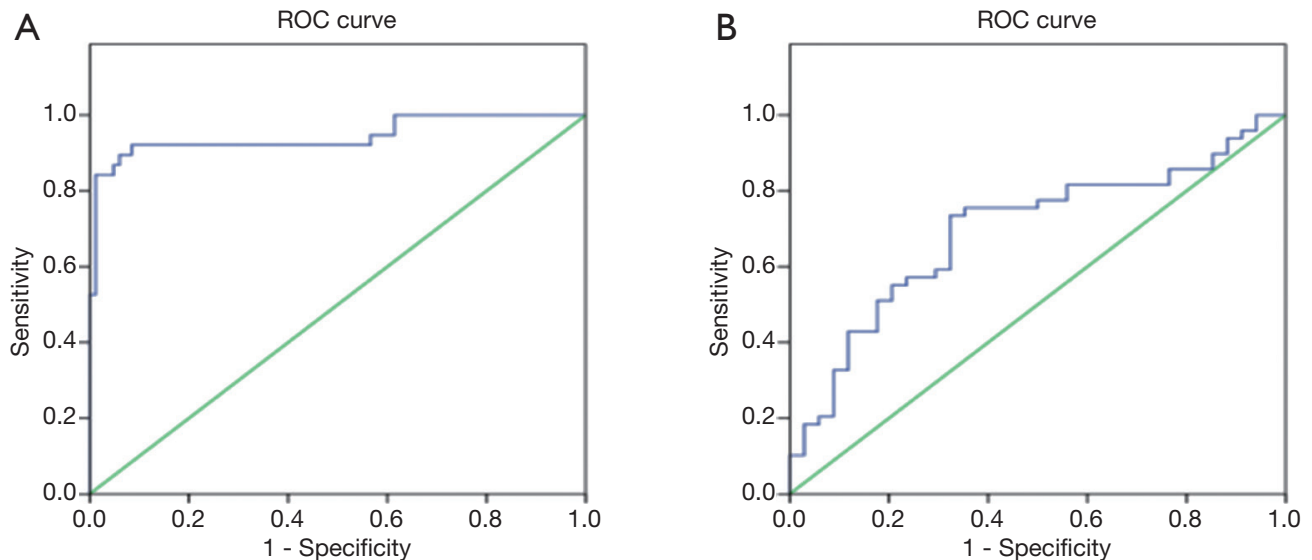

Figure 3 ROC curve analysis of biomarker SAP130. (A) The derived area under the ROC (AUC) curve between IPF patients and healthy control subjects was 0.944 (95\% CI, 0.810-0.997; cut-off $643.87 \mathrm{pg} / \mathrm{mL} ; 92.1 \%$ sensitivity; 69.9\% specificity). (B) The AUC of the ROC curve for the diagnosis of stable IPF versus AE-IPF diagnoses was 0.694 (95\% CI, 0.580-0.809) for SAP130 (95\% CI, 0.81-0.997; cut-off $741.46 \mathrm{pg} / \mathrm{mL} ; 63.3 \%$ sensitivity; 67.6\% specificity). SAP130, spliceosome-associated protein 130; ROC, receiver operating characteristic curve; AUC, area under curve; AE-IPF, acute exacerbation of idiopathic pulmonary fibrosis.

correlated positively with those of fibrosis on HRCT $(\mathrm{r}=0.4164, \mathrm{P}=0.0029$, Figure $4 E)$ and KL-6 level $(\mathrm{r}=0.4564$, $\mathrm{P}=0.0010$, Figure $4 G$ ), and negatively with FEV1 (\% predicted) $(\mathrm{r}=-0.3562, \mathrm{P}=0.0120$, Figure $4 A)$ and DLCO (\% predicted) ( $\mathrm{r}=-0.5550, \mathrm{P}<0.0001$, Figure 4 C); however, there was no significant correlation between serum levels of SAP 130 and FVC (\% predicted) $(\mathrm{r}=-0.2341, \mathrm{P}=0.1054$, Figure $4 B)$, GGO on HRCT $(\mathrm{r}=0.2811, \mathrm{P}=0.0504$, Figure $4 F)$ and $6 \mathrm{MWD}(\mathrm{r}=-0.1289, \mathrm{P}=0.3775$, Figure $4 D)$. Furthermore, serum SAP130 in AE-IPF patients positively correlated with the overall extent of fibrosis $(\mathrm{r}=0.3735$, $\mathrm{P}=0.0296$, Figure $5 A$ ) and GGO $(\mathrm{r}=0.4697, \mathrm{P}=0.0051$, Figure $5 B)$ on CT scans as well as serum KL-6 ( $\mathrm{r}=0.5470$, $\mathrm{P}=0.0008$, Figure 5C). There was no correlation between serum SAP130 levels and sex, age, and smoking history in both stable IPF and AE-IPF groups (data not shown).

\section{Comparison between survival group and death group in AE-IPF}

Twenty-four patients with AE-IPF survived for the minimum 1-year follow-up period. Patients with AE-IPF were divided into survival and non-survival groups after 1 year of follow-up. The differences of patient characteristics, serum markers, and HRCT data between survivors and non-survivors are shown in Table 3. No significant differences were found in terms of age, gender, and history of smoking between survivors and non-survivors.
As shown in Table 3, the baseline levels of serum SAP130 in the non-survival group were significantly higher than those in the survival group $(\mathrm{P}=0.0249)$. The serum SAP130 levels were $826.1 \pm 58.56 \mathrm{pg} / \mathrm{mL}$ and $1,032 \pm 63.10 \mathrm{pg} / \mathrm{mL}$ in the non-survival and survival groups, respectively. The extent of GGO and fibrosis in the non-survival group was significantly higher than that in the survival group $(20.93 \% \pm 2.279 \%$ vs. $15.00 \% \pm 1.645 \%, \mathrm{P}=0.0378$; $25.41 \pm 1.954$ vs. $18.86 \pm 1.182, \mathrm{P}=0.0032$, respectively).

\section{Discussion}

To our knowledge, this is the first study that revealed the clinical significance of SAP130 in the systemic and local lung compartments among IPF patients. We found a novel association between SAP130 levels and IPF disease. Significantly increased serum levels of SAP130 were detected in IPF patients. In addition, lung tissue from IPF patients exhibited significant SAP130 expression in active fibrotic and injury front-line areas versus controls. DLCO was considered to be a surrogate and objective marker for determining impairment of lung function and monitoring and quantifying disease progression. SAP130 levels in IPF negatively correlated with DLCO and FEV1, indicating that patients with higher SAP130 levels had more severe disease. Moreover, serum SAP130 levels in patients with stable conditions showed positive correlations with the extent of fibrosis on HRCT. Correlation analysis between 

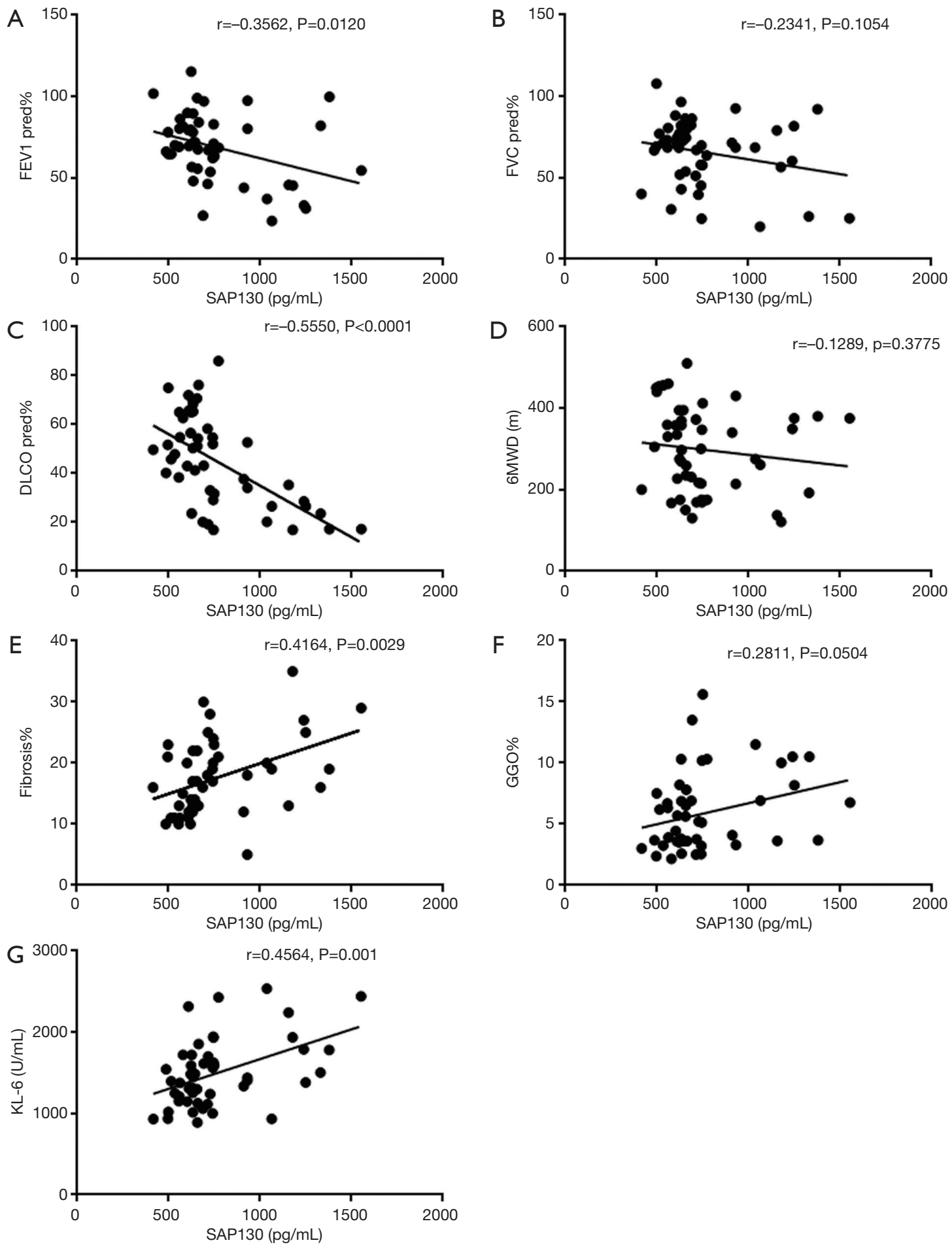

Figure 4 Correlations between serum SAP130 levels and clinical variables in patients with stable IPF. Correlations between serum SAP130 levels and (A) FEV1 (\% predicted); (B) FVC (\% predicted); (C) DLCO (\% predicted); (D) 6MWD; (E) Fibrosis Score (\%); (F) GGO Score (\%); (G) serum KL-6 were shown. SAP130, spliceosome-associated protein 130; IPF, idiopathic pulmonary fibrosis; FEV1, forced expiratory volume in 1; FVC, forced vital capacity; DLCO, carbon monoxide diffusing capacity; 6MWD, 6-minute walk distance; GGO, ground glass opacification; KL-6, Krebs von den Lungen 6. 
A
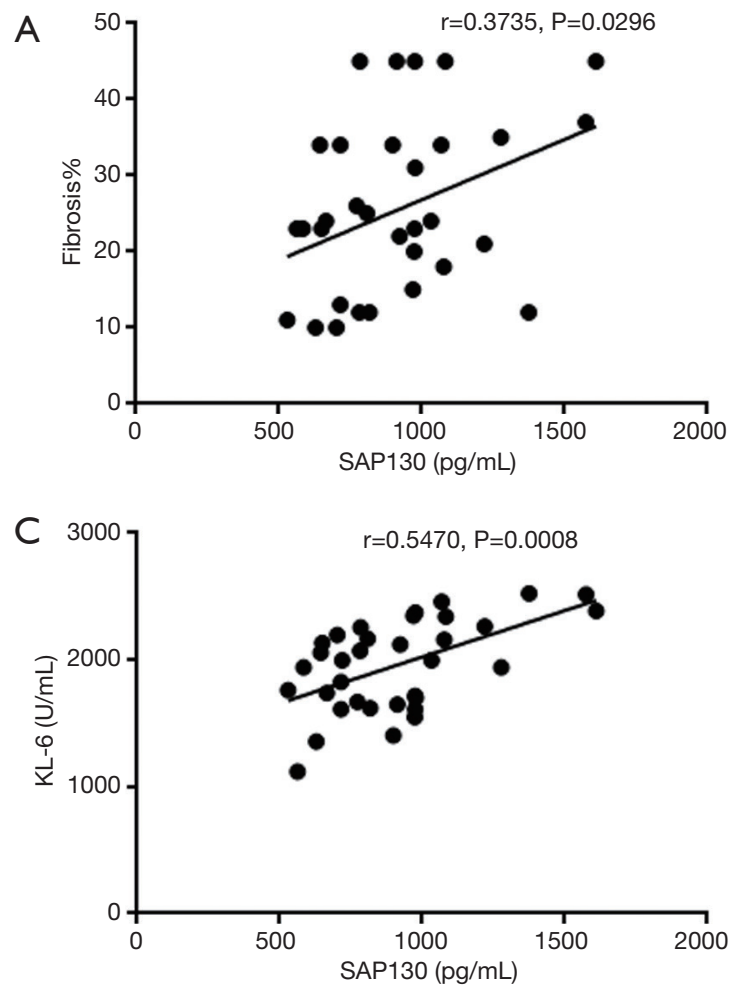

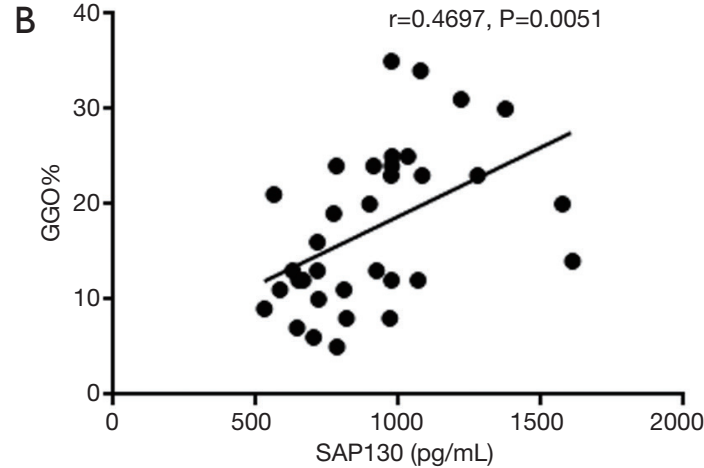

Figure 5 Correlations between serum SAP130 levels and clinical variables in patients with AE-IPF. Correlations between serum SAP130 levels and (A) Fibrosis Score (\%); (B) GGO Score (\%); (C) serum KL-6 were shown. SAP130, spliceosome-associated protein 130; AE-IPF, acute exacerbation of idiopathic pulmonary fibrosis; GGO, ground glass opacification; KL-6, Krebs von den Lungen 6.

Table 3 Comparison of baseline characteristics between non-survivors and survivors in patients with AE-IPF

\begin{tabular}{lccc}
\hline Characteristics & Survivor $(\mathrm{n}=20)$ & Non-survivor $(\mathrm{n}=14)$ & \\
\hline Men/women & $16 / 4$ & $10 / 4$ & 0.562 \\
Age, years (mean $\pm \mathrm{SD})$ & $66.40 \pm 1.56$ & $69.43 \pm 1.03$ & 0.150 \\
Smoking status & & & \\
Never-smoker & 5 & 11 & 0.447 \\
Former smoker & 15 & 1 & 0.809 \\
Current smoker & 0 & & 0.225 \\
CT visual score & & $20.93 \pm 2.28$ & 0.038 \\
GGO Score (\%) & $15.00 \pm 1.65$ & $25.41 \pm 1.95$ & 0.003 \\
Fibrosis Score $(\%)$ & $18.86 \pm 1.18$ & $1,032 \pm 63.10$ & 0.025 \\
SAP130 $(\mathrm{pg} / \mathrm{mL})$ & $826.1 \pm 58.56$ & & \\
\hline
\end{tabular}

AE-IPF, acute exacerbation of idiopathic pulmonary fibrosis; GGO, ground glass opacification; SAP130, spliceosome-associated protein 130.

SAP130 and 6MWT results did not show a relationship, which may be ascribed to the subjectivity of patient-related symptoms affecting $6 \mathrm{MWT}$ results. Unfortunately, this study did not demonstrate an excellent efficacy of SAP130 to diagnose IPF due to the low specificity. In fact, the diagnostic tool for IPF is expected to be highly specific. 
Most patients with AE-IPF simply cannot complete physiological tests such as PFT and 6MWT. Additionally, limited reproducibility and interobserver and intrapatient variability of the physiological tests commonly exist. Therefore, biomarkers that facilitate early and confident diagnosis, disease classification, estimation of the risk factor and prognosis, and dynamic evaluation of therapy response are especially urgently needed for AE-IPF patients. Our study showed that not only the extent of fibrosis but also the extent of GGO on high-resolution CT was associated with serum SAP130 levels in patients with AE-IPF. The new extensive GGO area may be rich in inflammatory factors where the alveoli are being destroyed, which should raise the possibility of acute exacerbation $(18,19)$. Extensive alveolar epithelial cell injury and cellular inflammation are due to multiple interactions of the cytokine and chemokine networks involved in the pathogenesis of AE-IPF. AE is an acute state of inflammation leading to a different protein expression pattern. Increased degree of lung epithelial injury during AE-IPF has previously been suggested and is supported by our finding of elevated serum SAP130 levels in AE-IPF as compared with those in the clinically stable phase. Previous studies revealed that the severity and rapidity of diffuse alveolar damage in lungs was correlated with the subsequent increase in serum KL-6 level (20,21). The short-term mortality in patients with AE-IPF in the current study is in line with previous studies. SAP130 as well as KL-6 were higher in non-survival patients with AE-IPF. Because the follow-up duration was too short to evaluate long-term clinical endpoints, we could not determine the true relationship between SAP130 levels and long-term prognosis. Our data suggested that elevated serum SAP130 was associated with different phases and the disease severity of IPF, which were likely involved in the pathogenesis of both stable IPF and AE-IPF.

Given the lack of consensus-based biomarkers for characterization of disease severity in IPF, our study added a novel and potential biomarker. Repeated or persistent epithelial injury is an inducer of proinflammatory and profibrotic factors that orchestrate lung fibrosis (21). The serum SAP130 was mainly derived from alveolar epithelial cells as our current study showed. SAP130 expression was enhanced in alveolar epithelia adjacent to mature fibrotic lesions in IPF, but not in controls. Biomarkers of alveolar epithelial cell damage and dysfunction have been studied for their diagnostic and predictive values in IPF. KL-6 is a mucin-like glycoprotein expressed on the surface membrane of alveolar epithelial cells. When alveolar epithelial cells proliferate and are activated or injured, KL-6 is released into the bloodstream (22). Elevated SAP130 levels in IPF patients may also reflect alveolar epithelial cells damage, injury, or necrosis, which is highly likely to act as a DAMP involved in lung fibrosis. Considering that the SAP130 protein can be secreted into the extracellular space, damaged alveolar tissue in fibrotic lungs is a potential main source of serum SAP130 in IPF patients. High levels of SAP 130 eventually enter the bloodstream through the damaged alveoli, which is in line with other epithelial DAMPs also released by dying or damaged alveolar epithelial cells for IPF, including KL-6, interleukin-25, interleukin-33, and thymic stromal lymphopoietin $(23,24)$.

Although the detailed biological function of SAP130 in non pathogen-mediated lung inflammation in clinical or preclinical models has not been fully elucidated, recent studies have revealed that SAP130 is involved and is upregulated in several other inflammatory conditions (25-27). SAP130 has been reported to contribute to alcohol-induced liver injury by inducing inflammasome-mediated production of inflammatory cytokines (25). High cytoplasmic SAP130 and extra-cellular release of SAP130 was observed in cultured neurons under hypoxia (25). Mincle/SAP30 signaling activation during subarachnoid hemorrhage initiates the Syk/CARD9/IL-1 $\beta$ pathway, leading to enhancement of neuronal necroptosis and microglial inflammation (26). An ongoing study by our group has demonstrated that SAP130 functions as a proinflammatory DAMP in lung fibrosis. We have demonstrated that alveolar epithelial cells exposed to bleomycin in a mouse model exhibited enhanced expression of SAP130. Studies in vitro and in vivo demonstrate that SAP130 has a detrimental role in the disease process of IPF. Inhibition of SAP130 in the central nervous system showed promising preclinical results by preventing and reducing ameliorating autoimmune encephalomyelitis (27). Admittedly, detailed studies concerning the role of SAP130 in the pathogenesis of IPF should be needed.

Several limitations in this study should be noted. First, the sample size of our study was too small with inherently reduced statistical power. Serum SAP130 in IPF patients was only measured once. Serial and dynamical measurements could provide deep insight into the clinical course and therapy response. Future studies in a longitudinal setting of larger sample sizes will further be performed to investigate whether serum levels of SAP130 are associated with disease progression and prognosis. Second, we did not evaluate the usefulness of SAP130 in the differential 
diagnosis of IPF with other fibrotic lung diseases. We cannot overstate its diagnostic value. Presumably other fibrotic lung diseases can cause an increase to varying degrees in serum 130 levels according to the mechanism of SAP130 release. Therefore, a large cohort study including other histological patterns of interstitial lung disease will be required to confirm the potential role of SAP130 as a differential biomarker in IPF.

\section{Conclusions}

In conclusion, we showed that SAP130 is elevated in the circulation and lungs of IPF patients. Circulating SAP130 levels may be reflective of IPF disease activity and severity. Our study provided preliminary evidence that SAP130 might be involved in the pathogenesis of IPF in both the clinically stable phase and upon acute exacerbation. Future prospective multi-center studies and expanded numbers of cases should examine and validate the diagnostic, prognostic and predictive value in the management of IPF patients. Additionally, further study is warranted to investigate the exact mechanism in which SAP130 contributes to the pathogenesis of IPF.

\section{Acknowledgments}

Funding: The study was supported by grants from the National Natural Science Foundation of China (81570066 and 81630001), Shanghai Top-Priority Clinical Key Disciplines Construction Project (2017ZZ02014), Shanghai Municipal Key Clinical Specialty (shslczdzk02202), Shanghai Shenkang Hospital Development Center Clinical Science and Technology Innovation Project (SHDC12018102), and Innovative Research Team of Highlevel Local Universities in Shanghai.

\section{Footnote}

Reporting Checklist: The authors have completed the STARD reporting checklist. Available at http://dx.doi.org/10.21037/ atm-20-4404

Data Sharing Statement: Available at http://dx.doi. org/10.21037/atm-20-4404

Conflicts of Interest: All authors have completed the ICMJE uniform disclosure form (available at http://dx.doi. org/10.21037/atm-20-4404). The authors have no conflicts of interest to declare.
Ethical Statement: The authors are accountable for all aspects of the work in ensuring that questions related to the accuracy or integrity of any part of the work are appropriately investigated and resolved. The study was conducted according to the principles of the Declaration of Helsinki (as revised in 2013). The protocol of this study was approved by the Ruijin Hospital Ethics Committee Shanghai Jiao Tong University School of Medicine, Human Ethics Review Committee of Fuzhou Pulmonary Hospital, Ethics Review Committee of the Affiliated Hospital of Fujian Medical University, and Ethics Review Committee of Fujian Geriatric Hospital. Written informed consents were obtained from all study subjects or their lineal consanguinities prior to enrollment.

Open Access Statement: This is an Open Access article distributed in accordance with the Creative Commons Attribution-NonCommercial-NoDerivs 4.0 International License (CC BY-NC-ND 4.0), which permits the noncommercial replication and distribution of the article with the strict proviso that no changes or edits are made and the original work is properly cited (including links to both the formal publication through the relevant DOI and the license). See: https://creativecommons.org/licenses/by-nc-nd/4.0/.

\section{References}

1. Lederer DJ, Martinez FJ. Idiopathic pulmonary fibrosis. $\mathrm{N}$ Engl J Med 2018;378:1811-23.

2. Shaw J, Marshall T, Morris H, et al. Idiopathic pulmonary fibrosis: a holistic approach to disease management in the antifibrotic age. J Thorac Dis 2017;9:4700-7.

3. Collard HR, Ryerson CJ, Corte TJ, et al. Acute exacerbation of idiopathic pulmonary fibrosis. An international working group. Am J Respir Crit Care Med 2016;194:265-75.

4. Biondini D, Balestro E, Sverzellati N, et al. Acute exacerbations of idiopathic pulmonary fibrosis (AE-IPF): an overview of current and future therapeutic strategies. Expert Rev Respir Med 2020;14:405-14.

5. Sauler M, Bazan IS, Lee PJ. Cell death in the lung: the apoptosis-necroptosis axis. Annu Rev Physiol 2019;81:375-402.

6. Ellson CD, Dunmore R, Hogaboam CM, et al. Dangerassociated molecular patterns and danger signals in idiopathic pulmonary fibrosis. Am J Respir Cell Mol Biol 2014;51:163-8.

7. Das BK, Xia L, Palandjian L, et al. Characterization of a 
protein complex containing spliceosomal proteins SAPs 49, 130, 145, and 155. Mol Cell Biol 1999;19:6796-802.

8. Yamasaki S, Ishikawa E, Sakuma M, et al. Mincle is an ITAM-coupled activating receptor that senses damaged cells. Nat Immunol 2008;9:1179-88.

9. Chiffoleau E. C-type lectin-like receptors as emerging orchestrators of sterile inflammation represent potential therapeutic targets. Front Immunol 2018;9:227.

10. Gong W, Guo K, Zheng T, et al. Preliminary exploration of the potential of spliceosome-associated protein 130 for predicting disease severity in Crohn's disease. Ann N Y Acad Sci 2020;1462:128-38.

11. Raghu G, Remy-Jardin M, Myers JL, et al. Diagnosis of Idiopathic Pulmonary Fibrosis. An Official ATS/ERS/JRS/ ALAT Clinical Practice Guideline. Am J Respir Crit Care Med 2018;198:e44-68.

12. Raghu G, Rochwerg B, Zhang Y, et al. An Official ATS/ ERS/JRS/ALAT Clinical Practice Guideline: Treatment of Idiopathic Pulmonary Fibrosis. An Update of the 2011 Clinical Practice Guideline. Am J Respir Crit Care Med 2015;192:644.

13. du Bois RM, Albera C, Bradford WZ, et al. 6-Minute walk distance is an independent predictor of mortality in patients with idiopathic pulmonary fibrosis. Eur Respir J 2014;43:1421-9.

14. Culver BH, Graham BL, Coates AL, et al. Recommendations for a Standardized Pulmonary Function Report. An Official American Thoracic Society Technical Statement. Am J Respir Crit Care Med 2017;196:1463-72.

15. Best AC, Meng J, Lynch AM, et al. Idiopathic pulmonary fibrosis: physiologic tests, quantitative CT indexes, and CT visual scores as predictors of mortality. Radiology 2008;246:935-40.

16. Flaherty KR, Andrei AC, Murray S, et al. Idiopathic pulmonary fibrosis: prognostic value of changes in physiology and six-minute-walk test. Am J Respir Crit Care Med 2006;174:803-9.

17. Ley B, Elicker BM, Hartman TE, et al. Idiopathic pulmonary fibrosis: CT and risk of death. Radiology

Cite this article as: Liu K, Liu D, Feng Y, Zhang H, Zeng D, Liu Q, Qu J. Spliceosome-associated protein 130: a novel biomarker for idiopathic pulmonary fibrosis. Ann Transl Med 2020;8(16):986. doi: 10.21037/atm-20-4404
2014;273:570-9.

18. Fujimoto K, Taniguchi H, Johkoh T, et al. Acute exacerbation of idiopathic pulmonary fibrosis: highresolution CT scores predict mortality. Eur Radiol 2012;22:83-92.

19. Hirano C, Ohshimo S, Horimasu Y, et al. Baseline HighResolution CT Findings Predict Acute Exacerbation of Idiopathic Pulmonary Fibrosis: German and Japanese Cohort Study. J Clin Med 2019;8:2069.

20. Collard HR, Calfee CS, Wolters PJ, et al. Plasma biomarker profifiles in acute exacerbation of idiopathic pulmonary fibrosis. Am J Physiol Lung Cell Mol Physiol 2010;299:L3-7.

21. Ohshimo S, Ishikawa N, Horimasu Y, et al. Baseline KL-6 predicts increased risk for acute exacerbation of idiopathic pulmonary fibrosis. Respir Med 2014;108:1031-9.

22. Camelo A, Dunmore R, Sleeman MA, et al. The epithelium in idiopathic pulmonary fibrosis: Breaking the barrier. Front Pharmacol 2014;4:173.

23. Li D, Guabiraba R, Besnard AG, et al. IL-33 promotes ST2dependent lung fibrosis by the induction of alternatively activated macrophages and innate lymphoid cells in mice. J Allergy Clin Immunol 2014;134:1422-32.e11.

24. Majewski S, Szewczyk K, Białas AJ, et al. Epithelial alarmins in serum and exhaled breath in patients with idiopathic pulmonary fibrosis: a prospective one-year follow-up cohort study. J Clin Med 2019;8:1590.

25. Xie Y, Guo H, Wang L, et al. Human albumin attenuates excessive innate immunity via inhibition of microglial Mincle/Syk signaling in subarachnoid hemorrhage. Brain Behav Immun 2017;60:346-60.

26. He Y, Xu L, Li B, et al. Macrophage-inducible C-type lectin/spleen tyrosine kinase signaling pathway contributes to neuroinflammation after subarachnoid hemorrhage in rats. Stroke 2015;46:2277-86.

27. N'diaye M, Brauner S, Flytzani S, et al. C-type lectin receptors $\mathrm{Mcl}$ and Mincle control development of multiple sclerosis-like neuroinflammation. J Clin Invest 2020;130:838-52. 